\title{
Integral Quartic Cayley Graphs on Abelian Groups
}

\author{
A. Abdollahi \\ Department of Mathematics \\ University of Isfahan \\ Isfahan 81746-73441 \\ Iran
}

and School of Mathematics

Institute for Research in Fundamental Sciences (IPM)

P.O.Box: 19395-5746, Tehran, Iran.

\author{
E. Vatandoost \\ Department of Mathematics \\ University of Isfahan \\ Isfahan 81746-73441 \\ Iran \\ e.vatandoost@math.ui.ac.ir
}

a.abdollahi@math.ui.ac.ir

Submitted: Aug 12, 2010; Accepted: Mar 29 2011; Published: Apr 14, 2011

Mathematics Subject Classifications: 05C25; 05C50

\begin{abstract}
A graph is called integral, if its adjacency eigenvalues are integers. In this paper we determine integral quartic Cayley graphs on finite abelian groups. As a side result we show that there are exactly 27 connected integral Cayley graphs up to 11 vertices.
\end{abstract}

\section{Introduction and Results}

A graph is called integral if all the eigenvalues of its adjacency matrix are integers. The notion of integral graphs was first introduced by Harary and Schwenk in 1974 [13].

It is known that the number of non-isomorphic $k$-regular integral graphs is finite (See e.g. [10]). Bussemaker and Cvetković [8] and independently Schwenk [20], proved that there are exactly 13 connected cubic integral graphs. It is shown in [4] and [5] that there are exactly 263 connected integral graphs on up to 11 vertices.

Radosavljević and Simić in [19] determined all thirteen nonregular nonbipartite connected integral graphs with maximum degree four. Stevanović [22] determined all connected 4-regular integral graphs avoiding \pm 3 in the spectrum. A survey of results on integral graphs may be found in [6].

Omidi [17] identified integral graphs with at most two cycles with no eigenvalues 0 . Sander [18] proved that Sudoku graphs are integral. In [2] it is shown that the total number of adjacency matrices of integral graphs with $n$ vertices is less than or equal to $2^{\frac{n(n-1)}{2}-\frac{n}{400}}$ for a sufficiently large $n$. Let $G$ be a non-trivial group with the identity element 1 and let $S$ be a non-empty subset of $G \backslash\{1\}$ such that $S=S^{-1}:=\left\{s^{-1} \mid s \in S\right\}$. The 
Cayley graph of $G$ with respect to $S$ which is denoted by $\Gamma(S: G)$ is the graph with vertex set $G$ and two vertices $a$ and $b$ are adjacent if $a b^{-1} \in S$.

Klotz and Sander $[15]$ proved that $\Gamma\left(U_{n}: \mathbb{Z}_{n}\right)$ is integral, where $\mathbb{Z}_{n}$ is the cyclic group of order $n$ and $U_{n}$ is the subset of all elements of $Z_{n}$ of order $n$. W. So [21] characterizes integral graphs among circulant graphs. In [1] we determined integral cubic Cayley graphs.

In this paper we study integral quartic Cayley graphs on finite abelian groups. Our main results are the following.

Theorem 1.1 Let $G$ be an abelian group such that $\Gamma(S: G)$ is integral, 4-regular and connected for some $S \subseteq G$. Then

$$
|G| \in\{5,6,8,9,10,12,16,18,20,24,25,32,36,40,48,50,60,64,72,80,96,100,120,144\} .
$$

As a side result, we show that

Theorem 1.2 There are exactly 27 connected integral Cayley graphs up to 11 vertices.

\section{Preliminaries}

First we give some facts that are needed in the next section. Let $n$ be a positive integer. Then $B(1, n)$ denotes the set $\{j \mid 1 \leq j<n,(j, n)=1\}$. Let $\omega=e^{\frac{2 \pi i}{n}}$ and

$$
C(r, n)=\sum_{j \in B(1, n)} \omega^{j r}, \quad 0 \leq r \leq n-1 .
$$

The function $C(r, n)$ is a Ramanujan sum. For integers $r$ and $n,(n>0)$, Ramanujan sums have only integral values (See [16] and [23]).

First we give some facts that are needed in the next section.

Lemma 2.1 Let $\omega=e^{\frac{\pi i}{n}}$, where $i^{2}=-1$. Then

i) $\sum_{j=1}^{2 n-1} \omega^{j}=-1$

ii) If $l$ is even, then $\sum_{j=1}^{n-1} \omega^{l j}=-1$.

iii) If $l$ is odd, then $\sum_{j=1}^{n-1} \omega^{l j}+\omega^{-l j}=0$.

Proof. The proof is straightforward.

Lemma 2.2 Let $G$ be a finite group and $a \in G$. If $\chi$ is a linear character of $G$ and $o(a)=2$, then $\chi(a)= \pm 1$. 
Proof. We know that each linear character is a homomorphism. So $(\chi(a))^{2}=\chi\left(a^{2}\right)=$ $\chi(1)=1$. Hence $\chi(a)= \pm 1$.

Lemma 2.3 [3] Let $G$ be a finite group of order $n$ whose irreducible characters (over $\mathbb{C})$ are $\chi_{1}, \ldots, \chi_{h}$ with respective degree $n_{1}, \ldots, n_{h}$. Then the spectrum of the Cayley graph $\Gamma(S: G)$ can be arranged as $\Lambda=\left\{\lambda_{i j k} \mid i=1, \ldots, h ; j, k=1, \ldots, n_{i}\right\}$ such that $\lambda_{i j 1}=\ldots=\lambda_{i j n_{i}}$ (this common value will be denoted by $\lambda_{i j}$ ), and

$$
\lambda_{i 1}^{t}+\ldots+\lambda_{i n_{i}}^{t}=\sum_{s_{1}, \ldots, s_{t} \in S} \rho_{i}\left(\Pi_{l=1}^{t} s_{l}\right)
$$

for any natural number $t$.

Lemma 2.4 [14] Let $C_{n}$ be the cyclic group generated by a of order $n$. Then the irreducible characters of $C_{n}$ are $\rho_{j}\left(a^{k}\right)=\omega^{j k}$, where $j, k=0,1, \ldots, n-1$.

Lemma 2.5 [14] Let $G=C_{n_{1}} \times \cdots \times C_{n_{r}}$ and $C_{n_{i}}=\left\langle a_{i}\right\rangle$, so that for any $i, j \in\{1, \ldots, r\}$, $\left(n_{i}, n_{j}\right) \neq 1$. If $\omega_{t}=e^{\frac{2 \pi i}{n_{t}}}$, then $n_{1} \cdots n_{r}$ irreducible characters of $G$ are

$$
\rho_{l_{1} \ldots l_{r}}\left(a_{1}^{k_{1}}, \ldots, a_{r}^{k_{r}}\right)=\omega_{1}^{l_{1} k_{1}} \omega_{2}^{l_{2} k_{2}} \ldots \omega_{r}^{l_{r} k_{r}}
$$

where $l_{i}=0,1, \ldots, n_{i}-1$ and $i=1,2, \ldots, r$.

Lemma 2.6 (Lemma 2.6 of [1]) Let $G$ be a group and $G=\langle S\rangle$, where $S=S^{-1}$ and $1 \notin S$. If $a \in S$ and $o(a)=m>2$, then $\Gamma(S: G)$ has the cycle with $m$ vertices as $a$ subgraph.

Lemma 2.7 (Lemma 2.7 of [1]) Let $G=\langle S\rangle$ be a group, $|G|=n,|S|=2, S=S^{-1} \not \supset$ 1. Then $\Gamma(S: G)$ is an integral graph if and only if $n \in\{3,4,6\}$.

Lemma 2.8 (Lemma 2.9 of [1]) Let $G$ be the cyclic group $\langle a\rangle,|G|=n>3$ and let $S$ be a generating set of $G$ such that $|S|=3, S=S^{-1}$ and $1 \notin S$. Then $\Gamma(S: G)$ is an integral graph if and only if $n \in\{4,6\}$.

Lemma 2.9 Let $G_{1}$ and $G_{2}$ be two non-trivial abelian groups and $G=G_{1} \times G_{2}$ such that $\Gamma(S: G)$ is integral, $G=\langle S\rangle, S=S^{-1} \not \supset 1$ and $|S|=4$. If $S_{1}=\left\{s_{1} \mid\left(s_{1}, g_{2}\right) \in\right.$ $S$ for some $\left.g_{2} \in G_{2}\right\} \backslash\{1\}$, then $\Gamma\left(S_{1}: G_{1}\right)$ is a connected integral graph.

Proof. Since $S$ generates $G$ and $S=S^{-1} \not \ngtr 1$ with four elements, $S_{1}$ generates $G_{1}$, $S_{1}=S_{1}^{-1} \not \supset 1$ and $\left|S_{1}\right| \in\{1,2,3,4\}$. It is easy to see that if $\left|S_{1}\right|=1$, then $\left|G_{1}\right|=2$ and so $\Gamma\left(S_{1}: G_{1}\right)$ is the complete graph $K_{2}$ with two vertices which is an integral graph.

Let $\chi_{0}$ and $\rho_{0}$ be the trivial irreducible characters of $G_{1}$ and $G_{2}$, respectively. Let $\lambda_{i 0}$ and $\lambda_{i}$ be the eigenvalues of $\Gamma(S: G)$ and $\Gamma\left(S_{1}: G_{1}\right)$ corresponding to irreducible characters of $\chi_{i} \times \rho_{0}$ and $\chi_{i}$, respectively. By Lemma 2.3,

$$
\lambda_{i 0}=\sum_{\left(g_{1}, g_{2}\right) \in S}\left(\chi_{i} \times \rho_{0}\right)\left(g_{1}, g_{2}\right)
$$


We have the following cases:

Case 1: If $\left|S_{1}\right|=4$, then $\lambda_{i 0}=\lambda_{i}$. It follows that $\Gamma\left(S_{1}: G_{1}\right)$ is an integral graph.

Case 2: Let $\left|S_{1}\right|=3$ and suppose that

$$
S=\left\{(a, x),\left(a^{-1}, x^{-1}\right),(b, y),\left(b, y^{-1}\right)\right\} \text { or } S=\left\{(a, x),\left(a^{-1}, x^{-1}\right),(b, y),\left(1, y^{-1}\right)\right\},
$$

where $o(b)=2$. Then $\lambda_{i 0}=\lambda_{i}+\chi_{i}(b)$ or $\lambda_{i 0}=\lambda_{i}+1$, respectively. Since $2 \mid \chi_{i}(b)-\chi_{i}(1)$, $\chi_{i}(b)$ is integer and So $\Gamma\left(S_{1}: G_{1}\right)$ is an integral graph.

Case 3: Let $\left|S_{1}\right|=2$ and $S=\left\{(a, x),\left(a^{-1}, x^{-1}\right),(1, y),\left(1, y^{-1}\right)\right\}$. Then $\lambda_{i 0}=\lambda_{i}+2$ and so $\Gamma\left(S_{1}: G_{1}\right)$ is an integral graph.

Case 4: Let $\left|S_{1}\right|=2$ and $S=\left\{(a, x),\left(a^{-1}, x^{-1}\right),(a, y),\left(a^{-1}, y^{-1}\right)\right\}$. Then $G_{1}$ is a cyclic group and

$$
\lambda_{i 0}=2 \sum_{s_{1} \in S_{1}} \chi_{i}\left(s_{1}\right)=2 \lambda_{i} .
$$

Since $\lambda_{i 0} \in\{-4, \pm 3, \pm 2, \pm 1,0\}(i \neq 0), \lambda_{1}$ and $\lambda_{2} \in\{-2, \pm 3 / 2, \pm 1, \pm 1 / 2,0\}$. By Lemmas 2.3 and $2.4, \lambda_{1}=2 \cos \left(\frac{2 \pi}{n}\right)$ and $\lambda_{2}=2 \cos 2\left(\frac{2 \pi}{n}\right)$, where $\left|G_{1}\right|=n$. By using $\cos 2 x=$ $2 \cos ^{2} x-1$ we conclude that $\lambda_{1}^{2}=\lambda_{2}+2$. Hence $\left(\lambda_{1}, \lambda_{2}\right) \in\{(0,-2),(-1,-1),(1,-1)\}$. If $\left(\lambda_{1}, \lambda_{2}\right)=(1,-1)$, then $\cos \left(\frac{2 \pi}{n}\right)=\frac{1}{2}$. So $n=6$. By [1, Lemma 2.7], $\Gamma\left(S_{1}: G_{1}\right)$ is an integral graph.

If $\left(\lambda_{1}, \lambda_{2}\right)=(0,-2)$, then $\cos \left(\frac{2 \pi}{n}\right)=0$. So $n=4$. By [1, Lemma 2.7] $\Gamma\left(S_{1}: G_{1}\right)$ is an integral graph.

If $\left(\lambda_{1}, \lambda_{2}\right)=(-1,-1)$, then $\cos \left(\frac{2 \pi}{n}\right)=\frac{-1}{2}$. So $n=3$. By [1, Lemma 2.7], $\Gamma\left(S_{1}: G_{1}\right)$ is an integral graph.

Lemma 2.10 (Lemma 2.11 of [1]) Let $G$ be a finite non-cyclic abelian group and let $G=\langle S\rangle$, where $|S|=3, S=S^{-1}$ and $1 \notin S$. Then $\Gamma(S: G)$ is an integral graph if and only if $|G| \in\{4,8,12\}$.

Theorem 2.11 (Theorem 1.1 of [1]) There are exactly seven connected cubic integral Cayley graphs. In particular, for a finite group $G$ and a subset $S=S^{-1} \not \supset 1$ with three elements, $\Gamma(S: G)$ is integral graph if and only if $G$ is isomorphic to one the following groups: $C_{2}^{2}, C_{4}, C_{6}, S_{3}, C_{2}^{3}, C_{2} \times C_{4}, D_{8}, C_{2} \times C_{6}, D_{12}, A_{4}, S_{4}, D_{8} \times C_{3}, D_{6} \times C_{4}$ or $A_{4} \times C_{2}$.

We denote as usual the complete graph on $n$ vertices by $K_{n}$ and the complete bipartite graph with parts of sizes $m$ and $n$ by $K_{m, n}$.

Lemma 2.12 There are exactly 40 connected, regular, integral graphs up to 10 vertices.

Proof. By [4], connected, regular, integral graphs are of type $\Gamma_{i}(i=1, \ldots, 40)$, where $\Gamma_{1}=K_{1}, \Gamma_{2}=K_{2}, \Gamma_{3}=K_{3}, \Gamma_{4}=K_{4}, \Gamma_{5}=K_{2,2}, \Gamma_{6}=K_{5}, \Gamma_{7}=K_{7}$ and connected, regular, integral graphs with $6,8,9$ and 10 vertices are displayed in tables 1,2,3 and 4 respectively. Graphs in these tables are represented in the form

$$
\Gamma_{i} a_{12} a_{13} a_{23} a_{14} a_{24} a_{34} \cdots a_{1 n} a_{2 n} \cdots a_{(n-1) n},
$$


where $\Gamma_{i}$ is the name of the corresponding integral graph and

$$
a_{12} a_{13} a_{23} a_{14} a_{24} a_{34} \cdots a_{1 n} a_{2 n} \cdots a_{(n-1) n}
$$

is the upper diagonal part of its adjacency matrix $\left[a_{i j}\right]_{n \times n}$ of the graph $\Gamma_{i}$. Also spectra of these graphs are displayed in tables $5,6,7$ and 8 respectively.

Lemma 2.13 Let $G=\langle a\rangle$ be a finite cyclic group of order $n>4$ and let $S$ be a generating set of $G$ such that $|S|=4, S=S^{-1}$ and $1 \notin S$. Then there exist two relatively prime, positive integers $r, s<n / 2(r \neq s)$ such that $S=\left\{a^{r}, a^{-r}, a^{s}, a^{-s}\right\}$.

Proof. Since $G$ is cyclic, $G$ has at most one element of order 2. Therefore $S$ cannot contain elements of order 2 as $|S|=4$ and $S=S^{-1}$. Since $a^{-\ell}=a^{n-\ell}$ for any integer $\ell$, it follows that there exist two positive integers $r, s<n / 2(r \neq s)$ such that $S=\left\{a^{r}, a^{-r}, a^{s}, a^{-s}\right\}$. Since $S$ generates $G, \operatorname{gcd}(r, s)=1$. This completes the proof.

Lemma 2.14 Let $G=\langle a\rangle$ be a finite cyclic group of order $n>4$ and let $S$ be a generating set of $G$ such that $|S|=4, S=S^{-1}$ and $1 \notin S$. Then $\Gamma(S: G)$ is integral if and only if one the following holds:

$$
\begin{aligned}
& \text { 1. } n=5 \text { and } S=\left\{a, a^{-1}, a^{2}, a^{-2}\right\} ; \\
& \text { 2. } n=6 \text { and } S=\left\{a, a^{-1}, a^{2}, a^{-2}\right\} ; \\
& \text { 3. } n=8 \text { and } S=\left\{a, a^{-1}, a^{3}, a^{-3}\right\} ; \\
& \text { 4. } n=10 \text { and } S=\left\{a, a^{-1}, a^{3}, a^{-3}\right\} ; \\
& \text { 5. } n=12 \text { and } S=\left\{a, a^{-1}, a^{5}, a^{-5}\right\} ; \\
& \text { 6. } n=12 \text { and } S=\left\{a^{2}, a^{-2}, a^{3}, a^{-3}\right\} \text {; } \\
& \text { 7. } n=12 \text { and } S=\left\{a^{4}, a^{-4}, a^{3}, a^{-3}\right\} \text {. }
\end{aligned}
$$

Proof. We need So's theorem [21, Theorem 7.1] and some knowledge about Euler's totient function $\varphi$ :

$$
\varphi(n)=2 \Longleftrightarrow n \in\{3,4,6\}, \quad \varphi(n)=4 \Longleftrightarrow n \in\{5,8,10,12\} .
$$

Let $\Gamma=\Gamma(S: G)$. According to So's theorem [21, Theorem 7.1] we have to consider two main cases.

Case 1. $\Gamma$ is a unitary Cayley graph.

Then the degree of regularity of $\Gamma$ is $\varphi(n)=4$, which implies $n \in\{5,8,10,12\}$. This gives graphs (1), (3), (4), (5) in the list of Lemma 2.14.

Case 2. It follows from Lemma 2.13 that there are proper divisors $r, s$ of $n, 1 \leq r<s<$ $n / 2$, such that

$$
S=\left\{a^{r}, a^{s}, a^{-r}, a^{-s}\right\}, \varphi\left(\frac{n}{r}\right)=2, \varphi\left(\frac{n}{s}\right)=2 \text { and } \operatorname{gcd}(r, s)=1 .
$$


This means that there is an integer $k$ such that $n=k r s$ and

$$
\frac{n}{r}=k s, \frac{n}{s}=k r \in\{3,4,6\} ; k, r, s \in\{1,2,3,4,6\} .
$$

We distinguish two subcases:

Case 2.1. $r=1$.

Then we have $\frac{n}{r}=n \in\{3,4,6\}$, which implies $n=6$.

Now $\frac{n}{s}=\frac{6}{s} \in\{3,4,6\}$ implies $s=2$. This gives graph (2).

Case 2.2. $r \geq 2$.

In this case only two subcases remain: $r=2, s=3$ and $r=3, s=4$. This leads to graphs (6) and (7) of the list.

To show that the determined graphs are not isomorphic, we need only care for the graphs on 12 vertices, i.e. graphs (5), (6), (7).

Graph (5) as a unitary Cayley graph of even order is bipartite, while (6) and (7) are not bipartite. Graph (7) contains a triangle. Graph (6) contains a circuit of length 5, but no triangle.

Corollary 2.15 Let $G=\langle a\rangle$ be a finite cyclic group of even order $n>4$. Let $S_{1}=$ $\left\{a^{r}, a^{-r}, a^{s}, a^{-s}\right\}$ and $S_{2}=\left\{a^{r}, a^{-r}, a^{s}, a^{-s}, a^{n / 2}\right\}$, where $r, s<n / 2(r \neq s), S_{t}=S_{t}^{-1} \not \ngtr 1$ and $G=\left\langle S_{t}\right\rangle$ for $t=1,2$. Then $\Gamma\left(S_{1}: G\right)$ is an integral graph if and only if $\Gamma\left(S_{2}: G\right)$ is an integral graph.

Proof. Let $\lambda_{j}$ and $\mu_{j} j \in\{0,1,2, \ldots, n-1\}$ be the eigenvalues of $\Gamma\left(S_{1}: G\right)$ and $\Gamma\left(S_{2}: G\right)$, respectively. By Lemmas 2.3 and $2.4, \lambda_{j}=\omega^{j r}+\omega^{-j r}+\omega^{j s}+\omega^{-j s}$ and $\mu_{j}=\omega^{j r}+\omega^{-j r}+\omega^{j s}+\omega^{-j s}+(-1)^{j}$ for $j \in\{0,1,2, \ldots, n-1\}$. This completes the proof.

Corollary 2.16 Let $G=\langle a\rangle$ be a finite cyclic group of order even $n>4$ and let $S$ be a generating set of $G$ such that $|S|=5, S=S^{-1}$ and $1 \notin S$. Then $\Gamma(S: G)$ is an integral graph if and only if one the following holds:

1. $n=6$ and $S=\left\{a, a^{-1}, a^{2}, a^{-2}, a^{3}\right\}$;

2. $n=8$ and $S=\left\{a, a^{-1}, a^{3}, a^{-3}, a^{4}\right\}$;

3. $n=10$ and $S=\left\{a, a^{-1}, a^{3}, a^{-3}, a^{5}\right\}$;

4. $n=12$ and $S=\left\{a, a^{-1}, a^{5}, a^{-5}, a^{6}\right\}$;

5. $n=12$ and $S=\left\{a^{2}, a^{-2}, a^{3}, a^{-3}, a^{6}\right\}$;

6. $n=12$ and $S=\left\{a^{4}, a^{-4}, a^{3}, a^{-3}, a^{6}\right\}$.

Proof. Note that $a^{n / 2} \in S$. Now Lemmas 2.13 and 2.14 complete the proof.

The following result follows from [21, Theorem 7.1]. 
Theorem 2.17 (Theorem 7.1 of [21]) Let $G$ be a finite group of order prime $p>1$. Then $\Gamma(S: G)$ is an integral graph if and only if $|S|=p-1$, where $S=S^{-1} \not \supset 1$ and $G=\langle S\rangle$.

Definition 2.18 A group $G$ is called Cayley simple if $\Gamma(S: G)$ is not integral, where $G=\langle S\rangle, 1 \notin S=S^{-1}$ and $S \neq G \backslash\{1\}$.

So by Theorem 2.17, we have

Corollary 2.19 Any finite group of prime order is Cayley simple.

Let us to put forward the following questions.

Question 2.20 Which finite groups are Cayley simple?

Question 2.21 Is any finite simple group, Cayley simple?

Lemma 2.22 There are exactly five connected, integral Cayley graphs with ten vertices.

Proof. We show that the graphs $\Gamma_{26}, \Gamma_{27}, \Gamma_{35}, \Gamma_{36}$ and $\Gamma_{37}$ are Cayley graphs and others are not (See table 4 ).

By table $8,|S| \in\{3,4,5,6,7,8,9\}$. By Theorem 2.11, the graphs $\Gamma_{38}, \Gamma_{39}$ and $\Gamma_{40}$ are not Cayley graphs.

It is clear that if $G$ is a finite group of order 10 and $|S|=9$, then $\Gamma(S: G)$ is the complete graph $K_{10}$ and so $\Gamma(S: G)=\Gamma_{26}$. Let $G$ be a finite group of order 10 . Then $G$ is isomorphic to $C_{10}$ or $D_{10}$. So we have the following two cases:

Case 1: Let $G=C_{10}=\langle a\rangle$.

If $|S|=4$ and $S=\left\{a, a^{3}, a^{7}, a^{9}\right\}$, then by easy calculations, one can see $\Gamma\left(S: C_{10}\right)$ is an integral graph with the spectrum $\left[-4,-1^{4}, 1^{4}, 4\right]$. Hence $\Gamma\left(S: C_{10}\right)=\Gamma_{37}$.

Let $|S|=5$. It is clear that $a^{5} \in S$. If $S=\left\{a, a^{3}, a^{5}, a^{7}, a^{9}\right\}$, then by a straightforward computation, one can see that $\Gamma\left(S: C_{10}\right)$ is an integral graph with the spectrum $\left[-5,0^{8}, 5\right]$. Thus $\Gamma\left(S: C_{10}\right)=\Gamma_{36}$.

Let $\Gamma\left(S: C_{10}\right)=\Gamma_{35}$. By table $8,|S|=5$ and so $a^{5} \in S$. It is clear that $S \neq$ $\left\{a, a^{3}, a^{5}, a^{7}, a^{9}\right\}$. By Lemmas 2.2 and 2.3, $\Gamma\left(S \backslash\left\{a^{5}\right\}: C_{10}\right)$ is an integral graph and so $\Gamma\left(S \backslash\left\{a^{5}\right\}: C_{10}\right)=\Gamma_{37}$. If $\chi$ is the irreducible character of $C_{10}$ corresponding to the eigenvalue 3 in graph $\Gamma\left(S: C_{10}\right)=\Gamma_{35}$, then by Lemmas 2.2 and 2.3 , the eigenvalue of $\Gamma\left(S \backslash\left\{a^{5}\right\}: C_{10}\right)$ corresponding to $\chi$ is 2 or 4 , which is impossible. Therefore $\Gamma_{35}$ is not a Cayley graph of $C_{10}$.

If $|S|=6$, then $a^{5} \notin S$ and so there is exactly one integer $r(1 \leq r \leq 4)$ such that $a^{r} \notin S$. Without loss of generality we can assume $r=4$ so that $S=\left\{a, a^{2}, a^{3}, a^{7}, a^{8}, a^{9}\right\}$. Then by a straightforward computation $\Gamma\left(S: C_{10}\right)$ is not an integral graph. Thus the graphs $\Gamma_{30}, \Gamma_{31}, \Gamma_{32}, \Gamma_{33}$ and $\Gamma_{34}$ are not Cayley graphs of $C_{10}$.

If $|S|=7$, then $a^{5} \in S$ and so there is exactly one integer $r \in\{1,2,3,4\}$ such that $a^{r} \notin S$. Without loss of generality we can assume $r=4$ so that $S=\left\{a, a^{2}, a^{3}, a^{5}, a^{7}, a^{8}, a^{9}\right\}$. Then by a straightforward computation $\Gamma\left(S: C_{10}\right)$ is not an integral graph. Thus the graphs 
$\Gamma_{28}$ and $\Gamma_{29}$ are not Cayley graphs of $C_{10}$.

If $|S|=8$, then $a^{5} \notin S$ and so $S=G \backslash\left\{1, a^{5}\right\}$. Now easy calculations show that $\Gamma\left(S: C_{10}\right)$ is an integral graph with the spectrum $\left[-2^{4}, 0^{5}, 8\right]$. Therefore $\Gamma\left(S: C_{10}\right)=\Gamma_{27}$.

Case 2: Let $G=D_{10}=\left\langle a, b \mid a^{5}=b^{2}=1,(a b)^{2}=1\right\rangle$.

If $|S|=4$ and $S=\left\{b, a b, a^{2} b, a^{3} b\right\}$, then by a straightforward computation, $\Gamma\left(S: D_{10}\right)$ is an integral graph with the the spectrum $\left[-4,-1^{4}, 1^{4}, 4\right]$. Therefore $\Gamma\left(S: C_{10}\right)=\Gamma_{37}$.

If $S_{1}=\left\{b, a b, a^{2} b, a^{3} b, a^{4} b\right\}$ and $S_{2}=\left\{a, a^{2}, a^{3}, a^{4}, b\right\}$, then $\Gamma\left(S_{1}: D_{10}\right)$ and $\Gamma\left(S_{2}: D_{10}\right)$ are integral graphs with the spectra $\left[-5,0^{8}, 5\right]$ and $\left[-2^{4}, 0^{4}, 3,5\right]$, respectively. Thus $\Gamma\left(S_{1}\right.$ : $\left.D_{10}\right)=\Gamma_{36}$ and $\Gamma\left(S_{2}: D_{10}\right)=\Gamma_{35}$.

Let $|S|=6$. Since $D_{10}$ has exactly two linear characters, it follows from Lemma 2.3 that $\Gamma\left(S: D_{10}\right)$ has exactly two simple eigenvalues. So the graphs $\Gamma_{30}, \Gamma_{31}$ and $\Gamma_{34}$ are not Cayley graphs of $D_{10}$. Since $|S|=6, S \cap\langle a\rangle=\langle a\rangle$ or $|S \cap\langle a\rangle|=2$. Suppose $\lambda$ be the eigenvalue of $\Gamma\left(S: D_{10}\right)$ corresponding to the linear character $\chi_{3}$ of $D_{10}$. Then by Lemma $2.3, \lambda=2$ if $S \cap\langle a\rangle=\langle a\rangle$ and $\lambda=-2$ if $|S \cap\langle a\rangle|=2$. On the contrary, let $\Gamma\left(S: D_{10}\right)=\Gamma_{32}$ or $\Gamma\left(S: D_{10}\right)=\Gamma_{33}$. Since $\operatorname{Spec}\left(\Gamma_{32}\right)=\left[-2^{5}, 1^{4}, 6\right]$ and $\operatorname{Spec}\left(\Gamma_{33}\right)=\left[-3,-2^{3}, 0^{2}, 1^{3}, 6\right]$, $|S \cap\langle a\rangle|=2$. Now if $\lambda_{11}$ and $\lambda_{12}$ are the eigenvalues of $\Gamma\left(S: D_{10}\right)$ corresponding to $\chi_{1}$, then by Lemma 2.3 and using the character table of $D_{10}, \lambda_{11}+\lambda_{12}=4 \cos \left(\frac{2 \pi}{5}\right)$ or $4 \cos \left(\frac{4 \pi}{5}\right)$. But the latter is not an integer, which is a contradiction. Hence $\Gamma_{32}$ and $\Gamma_{33}$ are not Cayley graphs of $D_{10}$.

Let $|S|=7$ and $\Gamma\left(S: D_{10}\right)=\Gamma_{28}$. Since $\lambda=-3$ is a simple eigenvalue of $\Gamma\left(S: D_{10}\right)=\Gamma_{28}$, it follows from Lemma 2.3 and the character table of $D_{10}$ that $\left\{b, a b, a^{2} b, a^{3} b, a^{4} b\right\} \subseteq S$. Thus $S=\left\{a, a^{-1}, b, a b, a^{2} b, a^{3} b, a^{4} b\right\}$ or $\left\{a^{2}, a^{-2}, b, a b, a^{2} b, a^{3} b, a^{4} b\right\}$. By easy calculations one finds that $\Gamma\left(S: D_{10}\right)=\Gamma_{28}$ is not integral graph, a contradiction. Therefore $\Gamma_{28}$ is not a Cayley graph of $D_{10}$.

Let $|S|=7$ and $\Gamma\left(S: D_{10}\right)=\Gamma_{29}$. Since $\lambda=1$ is a simple eigenvalue of $\Gamma\left(S: D_{10}\right)=\Gamma_{29}$, it follows from Lemma 2.3 and the character table of $D_{10}$ that $S \cap\langle a\rangle=\langle a\rangle$. If $\lambda_{k 1}$ and $\lambda_{k 2}$ are the eigenvalues of $\Gamma\left(S: D_{10}\right)$ corresponding to $\chi_{k}$ for $k \in\{1,2\}$, then Lemma 2.3 and the character table of $D_{10}$ implies that $\lambda_{11}+\lambda_{12}=\lambda_{21}+\lambda_{22}=-2$. Since the multiplicity 0 as an eigenvalue of $\Gamma\left(S: D_{10}\right)=\Gamma_{29}$ is $4, \lambda_{11}=0, \lambda_{12}=-2, \lambda_{21}=0$ and $\lambda_{22}=-2$ or $\lambda_{11}=-2, \lambda_{12}=0, \lambda_{21}=-2$ and $\lambda_{22}=0$ (Each one, two times). This shows that -2 is an eigenvalue of $\Gamma_{29}$, which is impossible. Therefore $\Gamma_{29}$ is not a Cayley graph of $D_{10}$.

Therefore there are exactly five connected, integral Cayley graphs with 10 vertices.

Lemma 2.23 There are exactly three connected, integral Cayley graphs with nine vertices.

Proof. We show that the graphs $\Gamma_{19}, \Gamma_{21}$ and $\Gamma_{24}$ are Cayley graphs and others are not (See table 3). It follows from table 7 that $|S| \in\{4,6,8\}$. Clearly if $G$ is a finite group of order nine and $|S|=8$, then $\Gamma(S: G)$ is the complete graph $K_{9}$ and so $\Gamma(S: G)=\Gamma_{19}$.

Let $G$ be a finite group of order 9 . Then $G$ is isomorphic to $C_{9}$ or $C_{3}^{2}$. We distinguish the following two cases:

Case 1: Let $G=C_{9}=\langle a\rangle$. If $|S|=4$, then by Lemma 2.14, $\Gamma\left(S: C_{9}\right)$ is not an integral graph. Since the graphs $\Gamma_{22}, \Gamma_{23}, \Gamma_{24}$ and $\Gamma_{25}$ are 4-regular integral graphs with 9 vertices, they are not Cayley graphs of $C_{9}$. 
Let $\Gamma\left(S: C_{9}\right)$ be an integral graph and $\lambda$ be the eigenvalue $\Gamma\left(S: C_{9}\right)$ corresponding to the irreducible character $\chi\left(a^{j}\right)=\omega^{j}$, where $|S|=6$ and $\omega=e^{\frac{2 \pi i}{9}}$. Since $\lambda$ and $\sum_{j=1}^{8} \omega^{j}$ are integers and $\omega^{r}+\omega^{-r}(r \neq 3)$ is not an integer, it follows from Lemma 2.3 that $S=\left\{a, a^{2}, a^{4}, a^{5}, a^{7}, a^{8}\right\}$. By an easy calculation one can see that $\Gamma\left(S: C_{9}\right)$ is an integral graph with the spectrum $\left[-3^{2}, 0^{6}, 6\right]$. Thus $\Gamma\left(S: C_{9}\right)=\Gamma_{21}$. This shows that $\Gamma_{20}$ is not a Cayley graph of $C_{9}$.

Case 2: Let $G=C_{3}^{2}=\langle b\rangle \times\langle b\rangle$. It is clear that $\omega+\omega^{2}=\omega^{2}+\omega^{4}=-1$, where $\omega=e^{\frac{2 \pi i}{3}}$.

If $|S|=4$, then by Lemmas 2.3 and 2.5 , all eigenvalues of $\Gamma\left(S: C_{3}^{2}\right)$ are in $\{-2,1,4\}$. Therefore $\Gamma_{22}, \Gamma_{23}$ and $\Gamma_{25}$ are not Cayley graphs of $C_{3}^{2}$. If

$$
S=\left\{(b, 1),\left(b^{2}, 1\right),(1, b),\left(1, b^{2}\right)\right\},
$$

then by a straightforward computation one can see that $\Gamma\left(S: C_{3}^{2}\right)$ is an integral graph with the spectrum $\left[-2^{4}, 1^{4}, 4\right]$. Hence $\Gamma\left(S: C_{3}^{2}\right)=\Gamma_{24}$.

If $|S|=6$, then by Lemmas 2.3 and 2.5 , all the eigenvalues of $\Gamma\left(S: C_{3}^{2}\right)$ are in $\{-3,0,6\}$. Thus $\Gamma_{20}$ is not a Cayley graph of $C_{3}^{2}$. If

$$
S=\left\{(b, 1),\left(b^{2}, 1\right),(1, b),\left(1, b^{2}\right),(b, b),\left(b^{2}, b^{2}\right)\right\},
$$

then $\Gamma\left(S: C_{3}^{2}\right)$ is an integral graph with the spectrum $\left[-3^{2}, 0^{6}, 6\right]$ and so $\Gamma\left(S: C_{3}^{2}\right)=\Gamma_{21}$. Therefore, the graphs $\Gamma_{19}, \Gamma_{21}$ and $\Gamma_{24}$ are the only Cayley graphs with nine vertices. This completes the proof.

Lemma 2.24 There are exactly six connected, integral Cayley graphs with eight vertices.

Proof. There are exactly six connected, regular integral graphs with eight vertices (See table 2). We show that these graphs are Cayley graphs. Clearly if $|S|=7$, then $\Gamma(S: G)$ is the complete graph $K_{8}$ and so $\Gamma(S: G)=\Gamma_{13}$.

Suppose $G=C_{8}=\langle a\rangle$ and

$$
S_{1}=\left\{a, a^{3}, a^{5}, a^{7}\right\}, S_{2}=\left\{a, a^{3}, a^{4}, a^{5}, a^{7}\right\}, S_{3}=\left\{a, a^{2}, a^{3}, a^{5}, a^{6}, a^{7}\right\} .
$$

Then $\Gamma\left(S_{1}: C_{8}\right), \Gamma\left(S_{2}: C_{8}\right)$ and $\Gamma\left(S_{3}: C_{8}\right)$ are integral graphs with the spectra $\left[-4,0^{6}, 4\right]$, $\left[-3,-1^{4}, 1^{2}, 5\right]$ and $\left[-2^{3}, 0^{4}, 6\right]$, respectively. Thus $\Gamma\left(S_{1}: C_{8}\right)=\Gamma_{17}, \Gamma\left(S_{2}: C_{8}\right)=\Gamma_{15}$ and $\Gamma\left(S_{3}: C_{8}\right)=\Gamma_{14}$.

Let $G=D_{8}=\left\langle a, b \mid a^{4}=b^{2}=1,(a b)^{2}=1\right\rangle, S_{1}=\left\{a, a^{3}, b\right\}$ and $S_{2}=\left\{a, a^{2}, a^{3}, b\right\}$. Then $\Gamma\left(S_{1}: D_{8}\right)$ and $\Gamma\left(S_{2}: D_{8}\right)$ are integral graphs with the spectra $\left[-3,-1^{3}, 1^{3}, 3\right]$ and $\left[-2^{3}, 0^{3}, 2,4\right]$, respectively. Hence $\Gamma\left(S_{1}: D_{8}\right)=\Gamma_{18}$ and $\Gamma\left(S_{2}: D_{8}\right)=\Gamma_{16}$.

Hence all of the connected, regular integral graphs with eight vertices are Cayley graphs. This completes the proof. 


\section{Proofs of Main Results}

In this section we prove our main results.

Proof of Theorem 1.1. Let $\Gamma(S: G)$ be integral. If $G$ is a cyclic group, then by Lemma 2.14, $n \in\{5,6,8,10,12\}$. Let $G$ be a finite abelian group, which is not cyclic. Suppose all of the elements of $S$ are of order two. Then $|G|=8$ or 16 . Otherwise since $S=S^{-1}$ and $1 \notin S$, the proof falls naturally into two parts.

i) There are exactly two elements of order two in $S$. Thus $G$ is isomorphic to $C_{m} \times C_{2}^{2}$. Let $S_{1}=\left\{s_{1} \in C_{m} \mid \exists x \in C_{2}^{2},\left(s_{1}, x\right) \in S\right\} \backslash\{1\}$. Since $\Gamma(S: G)$ is an integral graph, by Lemma 2.9, $\Gamma\left(S_{1}: C_{m}\right)$ is an integral graph. By Lemmas 2.7, 2.8 and 2.14, $m \in\{3,4,5,6,8,10,12\}$. Hence $n \in\{12,16,20,24,32,40,48\}$.

ii) There is no elements of order two in $S$. Thus $G$ is isomorphic to $C_{m_{1}} \times C_{m_{2}}$, where $\left(m_{1}, m_{2}\right) \neq 1$. Let $S_{1}=\left\{s_{1} \in C_{m_{1}} \mid \exists x \in C_{m_{2}},\left(s_{1}, x\right) \in S\right\} \backslash\{1\}$ and $S_{2}=\left\{s_{2} \in C_{m_{2}} \mid \exists x \in C_{m_{1}},\left(x, s_{2}\right) \in S\right\} \backslash\{1\}$. By Lemma 2.9, $\Gamma\left(S_{1}: C_{m_{1}}\right)$ and $\Gamma\left(S_{2}: C_{m_{2}}\right)$ are integral graphs. It follows from Lemmas 2.7, 2.8 and 2.14 that $m_{1}, m_{2} \in\{3,4,5,6,8,10,12\}$. Since $\left(m_{1}, m_{2}\right) \neq 1$, we have:

$n \in\{9,16,18,24,25,32,36,40,48,50,60,64,72,80,96,100,120,144\}$.

Proof of Theorem 1.2. It is easy to see that the graphs $\Gamma_{i}(1 \leq i \leq 8)$ are Cayley graphs.

Let $G=C_{6}=\langle a\rangle, S_{1}=\left\{a, a^{5}\right\}, S_{2}=\left\{a, a^{3}, a^{5}\right\}, S_{3}=\left\{a^{2}, a^{3}, a^{4}\right\}$ and $S_{4}=\left\{a, a^{2}, a^{4}, a^{5}\right\}$. Then $\Gamma\left(S_{1}: C_{6}\right), \Gamma\left(S_{2}: C_{6}\right), \Gamma\left(S_{3}: C_{6}\right)$ and $\Gamma\left(S_{4}: C_{6}\right)$ are integral with the spectra $\left[-2,-1^{2}, 1^{2}, 2\right],\left[-3,0^{4}, 3\right],\left[-2^{2}, 0^{2}, 1,3\right]$, and $\left[-2^{2}, 0^{3}, 4\right]$, respectively. Thus $\Gamma\left(S_{1}: C_{6}\right)=$ $\Gamma_{9}, \Gamma\left(S_{2}: C_{6}\right)=\Gamma_{10}, \Gamma\left(S_{2}: C_{6}\right)=\Gamma_{11}$ and $\Gamma\left(S_{3}: C_{6}\right)=\Gamma_{12}$. Hence all of the connected, regular integral graphs up to seven vertices are Cayley graphs. In other words there are exactly 12 connected, integral Cayley graphs up to seven vertices.

It follows from Lemmas 2.22, 2.23, 2.24 and Theorems 2.17 and 1.1, there are exactly 27 connected, integral Cayley graphs up to 11 vertices.

Table 1: Connected regular graphs with 6 vertices

\begin{tabular}{llllll}
\hline$\Gamma_{8}$ & 1 & 11 & 111 & 1111 & 11111 \\
$\Gamma_{9}$ & 1 & 01 & 001 & 0001 & 10001 \\
$\Gamma_{10}$ & 1 & 01 & 101 & 0101 & 10101 \\
$\Gamma_{11}$ & 1 & 11 & 001 & 0101 & 10011 \\
$\Gamma_{12}$ & 1 & 11 & 011 & 1011 & 11011 \\
\hline \hline
\end{tabular}


Table 2: Connected regular graphs with 8 vertices

\begin{tabular}{llllllll}
\hline$\Gamma_{13}$ & 1 & 11 & 111 & 1111 & 11111 & 111111 & 1111111 \\
$\Gamma_{14}$ & 1 & 10 & 011 & 1111 & 11110 & 111111 & 1111110 \\
$\Gamma_{15}$ & 1 & 10 & 110 & 1010 & 01111 & 111110 & 0111110 \\
$\Gamma_{16}$ & 1 & 10 & 010 & 1010 & 01110 & 101110 & 0101110 \\
$\Gamma_{17}$ & 1 & 10 & 100 & 1000 & 01111 & 011110 & 0111100 \\
$\Gamma_{18}$ & 1 & 10 & 010 & 0010 & 00011 & 100110 & 0110010 \\
\hline \hline
\end{tabular}

Table 3: Connected regular graphs with 9 vertices

\begin{tabular}{lllllllll}
\hline$\Gamma_{19}$ & 1 & 11 & 111 & 1111 & 11111 & 111111 & 1111111 & 11111111 \\
$\Gamma_{20}$ & 1 & 10 & 101 & 0110 & 01011 & 111111 & 1111110 & 11111100 \\
$\Gamma_{21}$ & 1 & 11 & 110 & 1100 & 10111 & 101110 & 0111111 & 01111110 \\
$\Gamma_{22}$ & 1 & 00 & 001 & 1100 & 00110 & 100111 & 0110110 & 11110000 \\
$\Gamma_{23}$ & 1 & 00 & 001 & 0011 & 11001 & 110010 & 0011011 & 11110000 \\
$\Gamma_{24}$ & 1 & 11 & 100 & 1001 & 01010 & 010011 & 0011010 & 00101011 \\
$\Gamma_{25}$ & 1 & 11 & 100 & 0010 & 01000 & 010111 & 0011110 & 10011100 \\
\hline \hline
\end{tabular}

Table 4: Connected regular graphs with 10 vertices

\begin{tabular}{llllllllll}
\hline$\Gamma_{26}$ & 1 & 11 & 111 & 1111 & 11111 & 111111 & 1111111 & 11111111 & 111111111 \\
$\Gamma_{27}$ & 1 & 11 & 111 & 1111 & 11110 & 111011 & 1101111 & 10111111 & 011111111 \\
$\Gamma_{28}$ & 1 & 00 & 001 & 1111 & 11111 & 111110 & 1111101 & 11110110 & 111101011 \\
$\Gamma_{29}$ & 1 & 00 & 001 & 1111 & 11110 & 111111 & 1111110 & 11110011 & 111111001 \\
$\Gamma_{30}$ & 1 & 11 & 111 & 1100 & 11001 & 001101 & 0011101 & 00111111 & 110011110 \\
$\Gamma_{31}$ & 1 & 11 & 000 & 0001 & 00011 & 111111 & 1111110 & 1111100 & 111111000 \\
$\Gamma_{32}$ & 1 & 11 & 110 & 1101 & 10110 & 101011 & 0111010 & 01101011 & 000111111 \\
$\Gamma_{33}$ & 1 & 00 & 110 & 1011 & 01110 & 111011 & 1110001 & 10111101 & 011111010 \\
$\Gamma_{34}$ & 1 & 10 & 010 & 0010 & 00011 & 111111 & 1111110 & 1111100 & 111111000 \\
$\Gamma_{35}$ & 1 & 10 & 101 & 1011 & 10111 & 011000 & 0101001 & 01001011 & 010001111 \\
$\Gamma_{36}$ & 1 & 10 & 100 & 1000 & 10000 & 011111 & 0111110 & 01111100 & 011111000 \\
$\Gamma_{37}$ & 1 & 10 & 100 & 1000 & 01110 & 011010 & 0101100 & 00111000 & 000001111 \\
$\Gamma_{38}$ & 0 & 10 & 010 & 1010 & 01010 & 001100 & 0000110 & 00000011 & 110000001 \\
$\Gamma_{39}$ & 0 & 00 & 000 & 1100 & 00111 & 001100 & 0011000 & 11000010 & 110000010 \\
$\Gamma_{40}$ & 1 & 10 & 100 & 0100 & 01000 & 001010 & 0010010 & 00011001 & 000101100 \\
\hline \hline
\end{tabular}


Spectra of connected, regular, integral graphs with $6,8,9$ and 10 vertices:

Table 5: Spectra of connected regular graphs with 6 vertices

\begin{tabular}{lllll}
\hline$\Gamma_{8}$ & & & 5 & $-1^{5}$ \\
$\Gamma_{9}$ & 2 & $1^{2}$ & $-1^{2}$ & -2 \\
$\Gamma_{10}$ & & 3 & $0^{4}$ & -3 \\
$\Gamma_{11}$ & 3 & 1 & $0^{2}$ & $-2^{2}$ \\
$\Gamma_{12}$ & & 4 & $0^{3}$ & $-2^{2}$ \\
\hline \hline
\end{tabular}

Table 6: Spectra of connected regular graphs with 8 vertices

\begin{tabular}{|c|c|c|c|c|}
\hline$\Gamma_{13}$ & & & 7 & $-1^{7}$ \\
\hline$\Gamma_{14}$ & & 6 & $0^{4}$ & $-2^{3}$ \\
\hline$\Gamma_{15}$ & 5 & $1^{2}$ & $-1^{4}$ & -3 \\
\hline$\Gamma_{16}$ & 4 & 2 & $0^{3}$ & $-2^{3}$ \\
\hline$\Gamma_{17}$ & & 4 & $0^{6}$ & -4 \\
\hline$\Gamma_{18}$ & 3 & $1^{3}$ & $-1^{3}$ & -3 \\
\hline
\end{tabular}

Table 7: Spectra of connected regular graphs with 9 vertices

\begin{tabular}{|c|c|c|c|c|c|c|c|c|}
\hline$\Gamma_{19}$ & & & & & & 8 & $-1^{8}$ & \\
\hline$\Gamma_{20}$ & & & & 1 & $0^{4}$ & $-2^{2}$ & -3 & \\
\hline$\Gamma_{21}$ & & & & & 6 & $0^{6}$ & $-3^{2}$ & \\
\hline$\Gamma_{22}$ & & & & 2 & $1^{2}$ & $-1^{2}$ & $-2^{3}$ & \\
\hline$\Gamma_{23}$ & 4 & & & 1 & $0^{2}$ & $-1^{2}$ & -2 & -3 \\
\hline$\Gamma_{24}$ & & & & & 4 & $1^{4}$ & $-2^{4}$ & \\
\hline$\Gamma_{25}$ & & & & $1^{3}$ & $0^{2}$ & $-2^{2}$ & -3 & \\
\hline
\end{tabular}


Table 8: Spectra of connected regular graphs with 10 vertices

\begin{tabular}{|c|c|c|c|c|c|c|c|}
\hline$\Gamma_{26}$ & & & & & & 9 & $-1^{9}$ \\
\hline$\Gamma_{27}$ & & & & & 8 & $0^{5}$ & $-2^{4}$ \\
\hline$\Gamma_{28}$ & & 7 & $1^{2}$ & $0^{2}$ & $-1^{2}$ & $-2^{2}$ & -3 \\
\hline$\Gamma_{29}$ & & & 7 & 1 & $0^{4}$ & $-1^{2}$ & $-3^{2}$ \\
\hline$\Gamma_{30}$ & 6 & 2 & 1 & $0^{2}$ & $-1^{2}$ & $-2^{2}$ & -3 \\
\hline$\Gamma_{31}$ & & & 6 & 2 & $0^{3}$ & $-1^{4}$ & -4 \\
\hline$\Gamma_{32}$ & & & & & 6 & $1^{4}$ & $-2^{5}$ \\
\hline$\Gamma_{33}$ & & & 6 & $1^{3}$ & $0^{2}$ & $-2^{3}$ & -3 \\
\hline$\Gamma_{34}$ & & 6 & $1^{2}$ & $0^{3}$ & $-1^{2}$ & -2 & -4 \\
\hline$\Gamma_{35}$ & & & & 5 & 3 & $0^{4}$ & $-2^{4}$ \\
\hline$\Gamma_{36}$ & & & & & 5 & $0^{8}$ & -5 \\
\hline$\Gamma_{37}$ & & & & 4 & $1^{4}$ & $-1^{4}$ & -4 \\
\hline$\Gamma_{38}$ & & & 3 & 2 & $1^{3}$ & $-1^{2}$ & $-2^{3}$ \\
\hline$\Gamma_{39}$ & 3 & 2 & $1^{2}$ & $0^{2}$ & $-1^{2}$ & -2 & -3 \\
\hline$\Gamma_{40}$ & & & & & 3 & $1^{5}$ & $-2^{4}$ \\
\hline
\end{tabular}

Character Table of $D_{2 n}, n=2 m+1$ odd

\begin{tabular}{llll}
\hline & 1 & $a^{r}$ & $b$ \\
\hline$\chi_{j}$ & 2 & $\omega^{j r}+\omega^{-j r}$ & 0 \\
$\chi_{m+1}$ & 1 & 1 & -1 \\
$\chi_{m+2}$ & 1 & 1 & 1 \\
\hline$\omega=e^{\frac{2 \pi i}{n}}, 1 \leq j \leq m$ and $1 \leq r \leq m$ & &
\end{tabular}

Acknowledgments. The authors are really indebted to the referee for many fruitful comments and for providing a computer-free proof of Lemma 2.14. This research was partially supported by the Center of Excellence for Mathematics, University of Isfahan. The first author's research was in part supported by the grant no. 88050040 from IPM.

\section{References}

[1] A. Abdollahi, E. Vatandoost, Which Cayley graphs are integral?, Electronic Journal of Combinatorics, 16 (2009) \#R122.

[2] O. Ahmadi, N. Alon, I.F. Blake, I.E. Shparlinski, Graphs with integral spectrum, Linear Algebra and its Applications, 430 (2009), 547-552.

[3] L. Babai, Spectra of Cayley graphs, Journal of Combinatorial Theory Ser. B, 27 (1979) 180-189.

[4] K. Balińska, D. Cvetković, M. Lepović, S. Simić, There are exactly 150 connected integral graphs up to 10 vertices, Univ. Beograd. Publ. Elektrotehn. Fak., Ser. Mat., 10 (1999) 95-105. 
[5] K.T. Balińska, M. Kupczyk, S.K. Simić, K.T. Zwierzyński, On generating all integral graphs on 11 vertices, The Technical University of Poznan, Computer Science Center Report No. 469, 1999/2000.

[6] K. Balińska, D. Cvetković, Z. Rodosavljevic, S. Simić, D. Stevanovic, A survey on integral graphs, Univ. Beograd, Publ. Elektrotehn. Fak. Ser. Mat. 13 (2003) 42-65.

[7] N. Biggs, Algebraic Graph Theory, Cambridge University Press, Cambridge, 1993.

[8] F.C.Bussemaker, D. Cvetković, There are exactly 13 connected, cubic, integral graphs., Univ. Beograd, Publ. Elektrotehn. Fak., Ser. Mat., Fiz., Nos. 544-576 (1976) $43-48$.

[9] D. M. Cvetković, M. Doob, H. Sachs, Spectra of graphs - Theory and applications, 3rd edition, Johann Ambrosius Barth Verlag, Heidelberg-Leipzig, 1995.

[10] D. Cvetković, Cubic integral graphs, Univ. Beograd, Publ. Elektrotehn. Fak., Ser. Mat., Fiz., Nos. 498-541 (1975) 107-113.

[11] H.D. Friedman, On the impossibility of certain Moore graphs, Journal of Combinatrial Theory Ser. B, 10 (1971) 245-252.

[12] J. Friedman, On Cayley graphs on the symmetric group generated by transpositions, Combinatorica, 20 (2000) 505-519.

[13] F. Harary, A.J. Schwenk, Which graphs have integral spectra?, Graphs and Combinatorics, 390 (1974) 45-51.

[14] G. James, M. Liebeck, Representations and Characters of groups, Cambridge University Press, Cambridge, 1993.

[15] W. Klotz, T. Sander, Some properties of unitary Cayley graphs, Electronic Journal of Combinatorics, 14 (2007) no. 1. Research Paper 45, 12 pp.

[16] P.J. McCarthy, Introduction to arithmetical functions, Universitext, Springer, New York, 1986.

[17] G.R. Omidi, On integral graphs with few cycles, Graphs and Combinatorics, 25 (2009) 841-849.

[18] T. Sander, Sudoku graphs are integral, Electronic Journal of Combinatorics, 16 (2009) \#N25.

[19] S. Simić, Z. Radosavljević, The nonregular, nonbipartite, integral graphs with maximum degree four. J. Comb., Inf. \& Syst. Sci., 20 1-4 (1995) 9-26.

[20] A.J. Schwenk, Exactly thirteen connected cubic graphs have integral spectra, Theory and applications of graphs (Proc. Internat. Conf., Western Mich. Univ., Kalamazoo, Mich., 1976), pp. 516-533, Lecture Notes in Math., 642, Springer, Berlin, 1978.

[21] W. So, Integral circulant graphs, Discrete Mathematics 306 (2006) 153-158.

[22] D. Stevanović, 4-Regular integral graphs avoiding \pm 3 in the spectrum, Univ. Beograd. Publ. Elektrotehn. Fak. Ser. Mat. 14 (2003) 99-110.

[23] S. Ramanujan, On certain trigonometrical sums and their applications in the theory of numbers, [Trans. Cambridge Philos. Soc. 22 (1918), no. 13, 259-276]. Collected papers of Srinivasa Ramanujan, 179-199, AMS Chelsea Publ., Providence, RI, 2000. 